


\section{PENSAR LA EDUCACIÓN EN BIOLOGÍA EN LOS NUEVOS ESCENARIOS SOCIALES: LA SINERGIA ENTRE MODELIZACIÓN, NATURALEZA DE LA CIENCIA, ASUNTOS SOCIO-CIENTÍFICOS Y MULTIRREFERENCIALIDAD}

\section{THINKING ABOUT BIOLOGY EDUCATION IN THE NEW SOCIAL SCENE: SYNERGY MODELLING,NATURE OF SCIENCE.SOCIOSCIENTIFIC ISSUES AND MULTIREFERENTIALITY}

Fecha de recepción: 12 de septiembre de 2014 Fecha de aprobación: 13 de noviembre de 2014

\section{Resumen}

La educación científica en biología se enfrenta en la actualidad a un conjunto de desafíos que nos obligan a repensar las metas de aprendizaje de nuestros alumnos y alumnas, en función de sus intereses y necesidades, y de la demanda social presente y orientada al futuro. Hoy se espera que los estudiantes adquieran una alfabetización científica que vaya más allá del conocimiento de contenidos propios de las disciplinas del área y estructurados según sus lógicas de construcción. En el mismo sentido el estudio comparativo internacional PISA (Programme for International Student Assessment) de la OECD (2006) sustenta una concepción de educación científica que parte de aquello que los estudiantes deben conocer, valorar y ser capaces de hacer para enfrentarse a situaciones de la vida real en el mundo actual y futuro. La nueva generación de reformas curriculares puestas en marcha en muchos países, refleja también un renovado interés por una educación científica basada en contextos, en sintonía con las aportaciones más recientes y significativas de la investigación internacional en el campo de Didáctica de las Ciencias, que abogan por una educación para la participación en sociedad, anclada en contextos relevantes de la vida real y basada en la adopción y comunicación de decisiones razonadas y documentadas respecto a asuntos socio-científicos. En consonancia con el giro en las demandas sociales a la educación científica y en biología, se hace necesario reconsiderar también la tarea de enseñar y nuestro rol como docentes. En el presente artículo se presenta un cierto consenso alcanzado en la Didáctica de las Ciencias y de la Biología, acerca de cuales son los desafíos que enfrentamos y cuáles las premisas teóricas para abordarlos. Estas últimas, surgidas de nuevas y fructíferas líneas de investigación en el campo. Se refiere especialmente a las que se ocupan de la contribución específica que las Ciencias Naturales pueden hacer a la formación de ciudadanía, a partir del abordaje de temas complejos en las aulas y de procesos de diseño de unidades didácticas basadas en la investigación en el marco de acciones de desarrollo profesional docente, a partir del análisis de algunos ejemplos de su aplicación en los ámbitos de la investigación didáctica y del desarrollo de materiales educativos.

\footnotetext{
1 Universidad Nacional de Río Negro. Argentina. Correo electrónico: nbahamonde@hotmail.com
} 


\section{Palabras clave}

Modelización, temas socio-científicos, educación alimentaria

\section{Summary}

Science education in biology is currently facing a number of challenges that require us to rethink the learning goals of our students, depending on their interests and needs and social demands present and future-oriented. Today we expect that students acquire scientific literacy that goes beyond its own content knowledge of the disciplines of the area and structured according to its logical construction. Similarly international comparative study PISA (Programme for International Student Assessment) of the OECD (2006) supports a conception of science education that starts from what students should know, should value and should be able to do to deal with real life situations in the present and future world. The new generation of curricular reforms implemented in many countries also reflect a renewed interest in science education based on contexts, in line with the most recent and significant contributions of international research in Science Education, Advocates for education for participation in society, anchored in relevant contexts of real life and based on the adoption and communication of reasoned and informed decisions regarding socio-scientific issues. In line with the shift in social demands to science education, it is also necessary to reconsider the task of teaching and our role as teachers. In this paper is presented a certain consensus in Science and Biology Education about what are the challenges to address and theoretical premises to face them, resulting from new and fruitful lines of research in the field. It refers especially to dealing with the specific contribution that natural sciences can make to the formation of citizenship, from tackling complex issues in classrooms and design processes of teaching units based on research and actions of teacher professional development, from analysis of some examples of its application in the fields of educational research and development of educational materials.

\section{Key Words}

Modelling, socio-scientific issues, nutrition education

\section{Introducción}

La educación científica en biología se enfrenta en la actualidad a un conjunto de desafíos que nos obligan a repensar las metas de aprendizaje de nuestros alumnos y alumnas, en función de sus intereses y necesidades, y de la demanda social presente y orientada al futuro. Hoy se espera que los estudiantes adquieran una alfabetización científica que vaya más allá del conocimiento de contenidos propios de las disciplinas del área y estructurados según sus lógicas de construcción. Por ejemplo, el estudio comparativo internacional PISA (Programme for International Student Assessment) de la OECD (2006) evaluó la alfabetización científica como área prioritaria por primera vez en el año 2006. "Como resultado de una considerable discusión el grupo de expertos de ciencias (del programa) desarrolló su propia definición de alfabetización científica: es la capacidad de usar el conocimiento científico para identificar preguntas y sacar conclusiones basadas en las pruebas, con la finalidad de entender y ayudar a tomar decisiones sobre el mundo natural y los cambios generados en él por la actividad humana" (Harlen, W, 2002). Así, la concepción de alfabetización científica que sustenta PISA parte de aquello que los estudiantes deben conocer, valorar y ser capaces de hacer para enfrentarse a situaciones de la vida real en el mundo actual y futuro. Está más centrada en el desarrollo de competencias, que en el aprendizaje de hechos y conceptos. Fensham (2009) analiza el uso de contextos de la vida real en el diseño de los ítemes de evaluación de Pisa y las implicancias que tiene este hecho en el renovado interés por una educación científica basada en contextos, puesta de manifiesto en la nueva generación de reformas curriculares en muchos países².

El enfoque de Pisa tiene también puntos de contacto con lo que Roberts (2007) denomina Visión II de la alfabetización científica, basada en el abordaje en las aulas de situaciones sociales con un componente científico que los estudiantes pueden encontrar en sus vidas y que habilite la toma de decisiones informada, en contraposición a la Visión I caracterizada por una mirada internalista de la ciencia y de sus productos: conceptos, leyes, teorías y procesos de investigación. Está en sintonía también con muchas de las aportaciones más recientes y significativas de la investigación internacional en el campo de Didáctica de las Ciencias, que abogan por una educación para la participación en sociedad, anclada en contextos relevantes de la vida real y basada en la adopción y comunicación de decisiones razonadas y documentadas respecto a asuntos científicos y tecnológicos con interés personal, cultural, profesional y social (Acevedo Díaz, 2010). Alineadas

2 La idea de introducir la enseñanza y el aprendizaje de las ciencias en contextos que fueran reales y significativos para los alumnos, fue explorada extensivamente a finales de los 80 , como respuesta al desafío "Science for all" (por e j. USA: National Science Foundation, 1983; Canada: Science Council of Canada, 1984; UK: The Royal Society, 1985)), aunque fue desplazada a mediados de los 90, cuando muchos proyectos curriculares en esos países definieron la ciencia que los estudiantes debían aprender en términos de largas listas de conceptos por ramas disciplinares (Fensham, 2009). 
con estas ideas, las demandas curriculares actuales de muchos países enfatizan la importancia de la adquisición de competencias para participar en asuntos socio-científicos a partir de juicios críticos (Jiménez Aleixandre y Federico-Agraso, 2009). En Argentina, el Ministerio de Educación propone garantizar una educación científica de calidad para todos, a partir de procesos de construcción de modelos científicos básicos por parte de los estudiantes, contextualizados en temas de relevancia y actualidad, así como de las formas de trabajo de la actividad científica, a partir del diseño y desarrollo de procesos de indagación escolary de actividades de promoción y valoración de la ciencia, con el propósito de que los estudiantes se impliquen en temas relacionados con ella y puedan interpretarla como una actividad humana de construcción colectiva, con historicidad, asociada a ideas, lenguajes y tecnologías específicas (Consejo Federal de Educación, Res. № 142/11). Estas definiciones se inscriben en lo que algunos autores denominan alfabetización científica funcional (Ziedler et al., 2005).

En consonancia con el giro en las demandas sociales a la educación científica y en biología, se hace necesario reconsiderar también la tarea de enseñar y nuestro rol como docentes. Según Abell (2000) conduce a plantearnos preguntas acerca de cómo los profesores de ciencias imaginan el futuro de sus estudiantes y qué conocimientos piensan que son críticos para su bienestar. También acerca de cómo creen que la ciencia se relaciona con otros modos de conocery de la forma en que los diferentes conocimientos sirven para dar sentido a las experiencias de la vida.

Para los profesores de ciencias puede no ser una tarea fácil cambiar sus prácticas tradicionales de enseñanza por nuevos formatos basados en contextos de la vida real que involucran cuestiones de ciencia y tecnología. Esto en parte puede explicarse porque su propia formación responde más a la ciencia académica que a la de sus áreas de aplicación. Los docentes pueden incluso sentirse inseguros sobre el conocimiento científico involucrado en esas cuestiones y con muchas dudas acerca de los aspectos no científicos relacionados (Fensham, 2009). Sin embargo, los nuevos escenarios sociales atravesados por retos complejos y multifacéticos, que involucran varias disciplinas y aspectos económicos, sociales, filosóficos y éticos (a los que nos referimos como asuntos socio-científicos) demandan cambios en la educación científica tradicional. Necesitamos diseñar nuevos escenarios educativos y nuevas formas de enseñar acordes con el mundo en que vivimos.

La discusión y la reflexión sobre estas cuestiones con los docentes abre la posibilidad de repensar con ellos el diseño y la puesta en marcha de la actividad científica en las aulas. "Los resultados de la investigación educativa refuerzan la idea que los docentes juegan un papel central en el diseño de los ambientes y experiencias de aprendizaje de sus alumnos. Los conocimientos de partida de los docentes y las decisiones que toman al planificar sus clases y convertirlas en actividades para el aula, pueden tener así un impacto decisivo en la comprensión de la ciencia y de los modelos científicos por parte de los alumnos. Conocer qué se proponen que hagan sus alumnos en las clases de ciencias y que conocimientos científicos necesitan para hacerlo, puede informar indirectamente sobre las relaciones que establecen entre los hechos del mundo y las explicaciones científicas y cómo las ponen en juego en los contextos específicos de las clases" (Bahamonde, 2009). Conscientes de los desafíos de la época y equipados con marcos teóricos renovados y propuestas de aplicación fundamentadas, los profesores y profesoras de ciencias podemos cambiar nuestras prácticas, desarrollando nuevas competencias orientadas al objetivo de una educación científica para la ciudadanía que incluya a todos los estudiantes.

En los próximos apartados presentaré, lo que considero cierto consenso alcanzado en la Didáctica de las Ciencias y de la Biología, acerca de cuales son los desafíos que enfrentamos y cuáles las premisas teóricas para abordarlos. Estas últimas, surgidas de nuevas y fructíferas líneas de investigación en el campo. Me referiré especialmente a las que se ocupan de la contribución específica que las Ciencias Naturales pueden hacer a la formación de ciudadanía a partir del abordaje de temas complejos en las aulas y de los procesos de diseño de unidades didácticas basadas en la investigación (Sanmartí, 2005) en el marco de procesos de desarrollo profesional docente (Bahamonde et al., 2014, Lozano et al. comunicación personal, 2014), a partir del análisis de algunos ejemplos de su aplicación en los ámbitos de la investigación didáctica y del desarrollo de materiales educativos.

\section{Sobre los desafíos de la educación científica y en Biología en la actualidad y algunas premisas teóricas para abordarlos}

Un primer desafío tiene que ver con la construcción por parte de los estudiantes de una visión actualizada sobre la ciencia y sobre los modelos científicos relevantes de cada disciplina, en este caso de la biología, que a la vez resulte funcional para ellos.

Creo que aquí surge una primera cuestión que nos interpela como educadores: ¿Qué ciencia enseñar? La investigadora en Didáctica de las Ciencias Mercè Izquierdo-Aymerich $(1999,2000)$ plantea que para poder enseñar ciencia resulta imprescindible contestar a la pregunta 
de qué es la ciencia. Podríamos responder que cualquier propuesta de enseñanza lleva implícita una determinada visión de ciencia que es necesario explicitar y analizar en función de los modelos epistemológicos vigentes. Abell (2000), plantea que se necesita aún más trabajo con los docentes para analizar y cuestionar las referencias monolíticas a la ciencia. Sin embargo, debemos considerar que los enfoques epistemológicos elegidos tienen que ser compatibles con los procesos de su enseñanza y aprendizaje, lo que nos lleva a descartar, por ejemplo, las posturas relativistas extremas. Así, los modelos de ciencia de realismo y racionalidad moderados, que superan las visiones empiristas clásicas, parecen ser más adecuados a la hora de pensar la educación científica. Desde estas perspectivas se concibe a los constructos de la ciencia, como representaciones sobre la realidad, es decir como modelos teóricos ajustados en diferente grado a los fenómenos (Giere, 1999). Estos modelos son potentes y generalizadores porque pueden ser aplicados a nuevas situaciones y comprobar que también funcionan y porque son útiles para predecir y tomar decisiones.

Enseñar ciencias, desde esta visión, es tender puentes que conectan los objetos y los hechos familiares o conocidos por los alumnos y las entidades conceptuales o modelos construidos por la ciencia para explicarlos. Es enseñar a los estudiantes a preguntarse y a pensar teóricamente sobre el mundo y favorecer en ellos procesos de autonomía creciente para seguir preguntándose, pensando y actuando en él. En este sentido hablamos de una ciencia que sea rigurosa y explicativa y, a la vez, funcional para los destinatarios.

Teniendo en cuenta la pregunta inicial y las ideas desarrolladas resulta ineludible la inclusión explícita en el diseño de la ciencia/biología escolar de actividades auxiliadas por la historia, la epistemología y la sociología de la ciencia, como herramientas para favorecer su comprensión como actividad humana que forma parte de la cultura y para entender los modos de construcción de conocimiento en el campo. Esto nos interpela como docentes. Según Adúriz-Bravo (2005):.. "Los profesores y profesoras de ciencias deberíamos ser capaces de realizar una exposición explícita y articulada de nuestra posición en torno a cuestiones tales cómo: cuál es el estatus de validez del conocimiento científico, de qué métodos se valen las ciencias para trabajar, cuáles son las características centrales de la explicación científica, cómo evoluciona el conocimiento científico lo largo del tiempo, o cuáles son las relaciones de la ciencia con la sociedad y la cultura de su época".

Recuperando esta idea, creemos que es necesario promover también el acercamiento de los científicos a las escuelas y de los estudiantes y docentes a los ámbitos científicos para favorecer una comprensión contextuada de la práctica social de referencia en circunstancias reales y tiempo presente.

Una segunda cuestión que lleva implícito este primer desafío es: ¿Cuáles son los modelos científicos escolares básicos y relevantes que tendríamos que enseñar? Hace tiempo, que como educadores sabemos, que no se puede enseñar todo y con el mismo nivel de profundidad. No se trata sólo de la imposibilidad de compatibilizar la enorme cantidad de conocimiento científico producido en el ámbito de la ciencia erudita con un horario escolar siempre escaso e insuficiente... Retomamos aquí la idea de que como docentes debemos cuestionarnos lo que enseñamos pensando en el sentido que debería tener para nuestros alumnos. En el caso de la enseñanza de la Biología, coincidimos con Izquierdo (1999) en considerar un principio de economía en la selección y secuenciación de los contenidos escolares. Pocos pero que expliquen muchos hechos, que ayuden a interpretar problemas distintos, a disponer de argumentos para decidir. Los programas y planificaciones deberían organizarse en un conjunto de núcleos temáticos relevantes desde lo social y pertinentes desde lo que hoy se considera conocimiento científico validado, articulados a partir de sus modelos teóricos básicos: Ser Vivo, Célula o Ecosistema, que agrupan preguntas, ideas, valores, experiencias y lenguaje. Esto es así porque como vimos, son irreductibles (no se reducen unos a otros) y, porque a partir de ellos, se derivan conocimientos estructurantes más generales, que dan sentido, por ejemplo, a conceptos como energía, estructura, cambio, unidad, diversidad (Izquierdo, 2005), vertebrando la educación científica.

Muchos autores han argumentado a favor de considerar el Modelo Ser Vivo como un modelo irreductible fundamental en el campo de la biología que debiera entonces enseñarse en las aulas (Weisz, 1975; Arca, Gudoni y Mazzoli, 1990; Cañal, 2003; Pujol, 2003; García, 2005, Gómez Galindo, 2007, entre otros, citado en Gómez Galindo, 2007). Tomando como base las ideas de García (2005), Gómez Galindo (2007) plantea que "el modelo de ser vivo puede considerarse como una teoría formada a su vez por tres familias de modelos: modelo "ser vivo-organismo ", modelo "servivo-ecosistema» y modelo «servivo-célula» en el marco de la Evolución. Este punto de vista es coherente con la definición de Giere (1999) del concepto de teoría y permite dar continuidad al currículo ya que posibilita el estudio de los seres vivos de forma articulada".

Sin embargo, es importante señalar que no se trata de enseñar los modelos en abstracto, sino de organizar la actividad científica escolar, alrededor de estos modelos 
o de algunos de sus aspectos. Nos referimos al diseño de situaciones o preguntas problematizadoras, motivadoras, contextuadas y significativas para los alumnos, relacionadas con los hechos y fenómenos que pueden explicarse a partir de las entidades conceptuales que estructuran el modelo de referencia, y que tienen potencial para interpretar y explicar otros hechos y fenómenos similares. Apuntamos a un cambio que promueve la contextualización de los temas y la modelización de los fenómenos.

Para citar un ejemplo, las problemáticas abiertas en los últimos 60 años en el campo de la biología molecular y genética han planteado nuevos e importantes desafíos científicos y debates sociales. Entendemos que es importante que estos debates entren a las aulas, favoreciendo procesos de enculturación científica, con el propósito de que los estudiantes se impliquen en problemas socio-científicos, construyan nociones científicas actualizadas que los habiliten al ejercicio de una ciudadanía de pleno derecho, informada y responsable y entiendan a la ciencia como una actividad humana de construcción colectiva e histórica, asociada a ideas, lenguajes y tecnologías específicas. Así, los temas de la agenda científica actual, que concitan el interés, la opinión y hasta la polémica en la sociedad, empujados a veces por intereses específicos desde los medios de comunicación como los vinculadas a la Biotecnología o la Bioética constituyen puertas de entrada ricas, para abordar problemas "auténticos" en el aula, revisitar y profundizar los modelos biológicos básicos y los contextos y escenarios de su construcción y favorecer, en simultáneo, el desarrollo de razonamiento ético y moral.

Un segundo desafío se relaciona con la necesidad de que los estudiantes logren una alfabetización avanzada, a partir del desarrollo y la mejora de competencias cognitivo lingüísticas específicas en el contexto de la ciencia/ biología escolar.

Esta meta a lograr nos conduce a la pregunta ¿Cuál es la relación entre ciencia escolar y lenguaje? y nos lleva a analizar las interacciones entre pensamiento, lenguaje y acción, en el marco de la actividad científica en el aula.

Hoy sabemos que hablar, leer y escribir ciencia contribuyen con aportes específicos e insustituibles al desarrollo de capacidades cognitivo lingüísticas y a la alfabetización de los estudiantes. Esto es así porque el lenguaje juega un rol irreemplazable en el proceso de construir la mirada científica, ya que permite darle nombre a las relaciones observadas cuando exploramos hechos y fenómenos, conectándolas con las entidades conceptuales que las justifican. Asimismo favorece la emergencia de nuevos significados, de nuevas palabras y nuevos argumentos, convirtiéndose en una herramienta para cambiar la forma de pensar el mundo (Lemke, 1997).

En ese marco son tan importantes las preguntas y los "experimentos" escolares, como las discusiones acerca de los resultados y sus interpretaciones y los textos que se escriben para comunicar y estructurar las nuevas ideas. La introducción de vocabulario científico va asociada a la comprensión de las ideas y conceptos que representan las palabras, alejándose de un lenguaje formal pero vacío de contenido. No se trata de aprender definiciones sino de poder explicar.

Enseñar ciencia a nuestros alumnos implica, entonces, promover la construcción de entidades conceptuales, en simultáneo con el desarrollo de habilidades instrumentales básicas de razonamiento y comunicación, es decir, leer y comprender, hablar, escribir y argumentar sobre ciencia. En ese contexto, modelizar fenómenos científicos en la escuela implica también aprender una combinación de géneros lingüísticos para dar a conocer el pensamiento y la acción. Por esta razón, la comprensión lectora y la producción de diferentes tipos de textos orales y escritos son habilidades cognitivo lingüísticas que es necesario contribuir a desarrollar en las clases de ciencia/biología, y herramientas para pensar y construir significados científicos. Una mención especial merece el papel de la argumentación en la educación científica. La argumentación puede estar involucrada en una variedad de situaciones de enseñanza asociadas a diferentes objetivos. Los tres principales que señalan Jiménez-Aleixandre y Erduran (2008) apuntan a que los alumnos: desarrollen conocimientos y habilidades sobre naturaleza de la ciencia; desarrollen la ciudadanía de los estudiantes, en particular en el caso de abordaje de asuntos socio-científicos y favorezcan el aprendizaje, más específicamente desarrollando un pensamiento de orden superior en situaciones particulares de argumentación.

Con el propósito de que los estudiantes desarrollen estas habilidades, la actividad científica escolar debería brindarles oportunidades para seleccionar e interpretar información proveniente de diversas fuentes, analizarla y organizarla utilizando distintos formatos. En el mismo sentido consideramos irreemplazable el trabajo con textos informativos o de divulgación y comunicación masiva, incluyendo los audiovisuales y multimediales, con contenido y lenguaje científico en los que se puedan identificar evidencias y calidad de los argumentos, pero también, con textos de ficción que retomen los problemas planteados y permitan ampliar el análisis, trascendiendo la mirada científica a través de la literatura, el cine, el humor 
gráfico, etcétera. Es importante que los alumnos puedan participar en una variedad de situaciones comunicativas orales o mediadas por las nuevas tecnologías y elaborar textos descriptivos y argumentativos para exponerlos y defenderlos ante una audiencia. En las clases de biología necesitamos incorporar otras formas de representación, por ejemplo, los dibujos, las maquetas, las fórmulas y los diagramas, ya que cada modo comunicativo enfatiza algún aspecto del modelo seleccionado. Algunos estudios muestran que la comprensión de los alumnos aumenta al utilizar varios modos comunicativos de forma relacionada, por ejemplo, explicar oralmente los dibujos, escribir un texto al lado de una maqueta, hacer un diagrama o una gráfica y explicarla, etcétera (Gómez Galindo, 2009).

Por lo general se supone que la capacidad de comunicar las propias ideas oralmente y por escrito, desarrollada en las clases de lengua, posibilitará a los estudiantes comunicarse en cualquier disciplina. Sin embargo, vemos en las clases de biología las dificultades que tienen para utilizar el lenguaje en contexto. Actividades como describir, comparar, justificar o argumentar utilizando pruebas al exponer o al escribir, forman parte de la enseñanza y el aprendizaje de las ciencias naturales. No se trata sólo de aprender los modelos científicos, sino de aprender también las formas de comunicar las interpretaciones que ha consensuado la comunidad científica acerca de los hechos y fenómenos de la naturaleza.

En nuestras sociedades basadas en la información es cada vez más importante, que podamos ayudar a los estudiantes a utilizar la información para construir conocimiento.

Un tercer desafío se vincula a la inclusión educativa y a la necesidad de brindar igualdad de oportunidades a todos los estudiantes para el aprendizaje de las ciencias. Nos enfrenta a la pregunta: ¿Cómo diseñar una ciencia/biología propia de la clase, accesible y motivadora, que tome en cuenta los saberes, experiencias e intereses de nuestros alumnos? Sabemos que muchos de ellos fracasan en sus estudios, y creo que por lo menos una parte de la responsabilidad nos corresponde a los docentes de ciencias, que, a veces, y tal vez de modo no consciente contribuimos a reforzar representaciones sociales deformadas acerca de la ciencia y los científicos, que desinteresan a los estudiantes.

Teniendo en cuenta este desafío, las clases de ciencia/ biología deberían brindar oportunidades para desmitificar la idea de que la ciencia es una materia difícil o accesible sólo a unas pocas personas. Hoy se considera que una educación científica es de calidad cuando lo es para todos. En este marco entendemos que no se puede privar a ningún estudiante del derecho a conocer un área de la cultura humana socialmente construida, las ciencias naturales, que proporciona elementos para comprender el mundo y situarse en él.

Para lograrlo, proponemos el diseño de ambientes de aprendizaje, ricos y potentes, que favorezcan en el aula diversas vías de acceso al conocimiento (histórica, artística, tecnológica...) y puentes que conecten los hechos y experiencias familiares o conocidos por los estudiantes con las entidades conceptuales construidas por la ciencia para explicarlos. El aula puede constituirse así en un espacio de diálogo e intercambio entre diversas formas de ver, de hablar y de pensar, en el que los participantes, alumnos y docente, ponen en juego las distintas representaciones que han construido sobre la realidad, para contrastarlas a través de exploraciones e interacciones directas con los seres vivos. Los hechos elegidos se plantean como problemas, preguntas o desafíos porque interpelan a los estudiantes sobre el funcionamiento del mundo, poniéndolos en la situación de buscar respuestas y elaborar explicaciones.

El propósito es que todos los alumnos se conviertan en protagonistas de la actividad científica escolar. Para concretarlo, entendemos que es importante tener en cuenta los puntos de partida y los tiempos de aprendizaje de cada alumno y elaborar y poner en marcha secuencias didácticas basadas en la investigación (Sanmartí, 2005) que integren actividades diversificadas y contemplen diferentes niveles de progresión en la construcción de los modelos.

Por último el desafío que entendemos integra y articula los anteriores, retomando las finalidades de una alfabetización científica funcional, se refiere al logro por parte de los estudiantes de una formación para el ejercicio de una ciudadanía de pleno derecho y la toma de decisiones informada y responsable, a partir del abordaje y la comprensión progresiva de problemas relevantes a nivel personaly social.

Creemos que este desafío exige de la educación en biología un compromiso claro y sostenido con la formación ciudadana, en el sentido de una formación que capacite para la acción. Muchos investigadores, desde líneas de trabajo diferentes pero a mi juicio complementarias, venimos planteando la necesidad de abordar problemas o temas complejos en las aulas vinculados tradicionalmente con el campo de la salud o el ambiente, a partir de una conceptualización abarcadora y multidimensional que articule, entre otras, la perspectiva científica, dando cuenta de su complejidad (Bahamonde, 2007; Bahamonde y Pujol 2009; Bahamonde y Diaco, 2013; Revel Chion, 2013). Varios autores en la comunidad internacional de didactas de la ciencia trabajan en líneas de argumentación convergentes, por ejemplo, en la idea de una educación en asuntos 
socio-científicos (Ziedler et al., 2005; Aikenhead, 2011; Sadler, 2011) que propicie el abordaje de contenidos de ciencia relevantes en el mundo cotidiano, y a menudo controversiales, destacando la importancia del aprendizaje situado, de una enseñanza que atienda los contextos y del desarrollo de razonamiento moral por parte de los estudiantes. Legardez y Simonneaux (2006), proponen la enseñanza de cuestiones socialmente vivas (QSV), refiriéndose a ellas como cuestiones de aprendizaje "donde se enfrentan los valores y los intereses" con una carga de emociones importante, "a menudo políticamente sensibles, intelectualmente complejas, y cuyos asuntos son importantes para el presente y para el futuro común" (Tutiaux-Guillon, 2006, citado en Legardez y Simonneaux, 2006). Christensen y Fensham (2012) plantean que las sociedades modernas signadas de manera muy significativa por la ciencia y la tecnología constituyen los escenarios para la vida de los ciudadanos actuales y del futuro. En vez de simplificarse estas sociedades están cada vez más preocupadas por temas que son complejos porque involucran incertidumbre y multidisciplinariedad.

La educación científica de los futuros ciudadanos no puede hacer caso omiso de los grandes desafíos que la ciencia y la tecnología imponen. Desde estas perspectivas teóricas la argumentación constituye un elemento central no sólo de una cultura científica de la ciudadanía, sino, y de manera más amplia, de una visión transdisciplinaria de la cultura, orientada a la comprensión de la interdependencia entre ciencia y sociedad. Que los estudiantes mejoren la argumentación sobre asuntos socio-científicos implica varios desafíos. Por un lado, al tratarse de temas potencialmente controversiales ofrecen oportunidades para la explicitación de diversos de puntos de vista y líneas argumentativas. Por otra parte, su naturaleza interdisciplinaria requiere a los estudiantes articular diferentes dominios. Finalmente, atendiendo la influencia de los medios de comunicación y los debates públicos sobre estos asuntos en la argumentación de los estudiantes, que dificultan el logro de discursos autónomos (Simonneaux, 2008), se requiere trabajar con ellos en la construcción de los propios puntos de vista, basados en conocimiento, ayudándolos a ser capaces de lidiar con la complejidad y la incertidumbre frente a la toma de decisiones.

En los próximos apartados me referiré a la línea de investigación que desarrollo y a algunas propuestas de aplicación derivadas, ya que considero que podrían constituir un aporte vinculado con el logro de este desafío.

\section{Modelizando nociones complejas a partir de la investigación / La puesta en marcha de investigaciones sobre modelización de nociones complejas}

La propuesta teórica que presento a continuación se inscribe en las preocupaciones planteadas. Fue desarrollada en el marco de dos investigaciones sobre los modelos de conocimiento escolar puestos en juego por docentes de distintos niveles educativos (infantil, primario y secundario) al planificar y enseñar en las aulas el tema de la alimentación humana (Bahamonde, 2007; Bahamonde y Pujol, 2009; Bahamonde y Diaco, 2013). Las investigaciones llevadas a cabo permitieron ampliar, profundizar y actualizar el marco teórico para el abordaje de la Educación Alimentaria con el aporte de referentes científicos de distintas disciplinas de los campos biológico y social. Se identificaron diferencias significativas entre esas visiones, que la conceptualizan como una temática compleja y multidimensional que requiere perspectivas interdisciplinarias y los modelos de conocimiento de los docentes, muchas veces limitados o parciales, signados principalmente por un enfoque nutricional-sanitarista y una tendencia reduccionista hacia la medicalización de la alimentación. Este enfoque se traducía en la uniformidad de las actividades que seleccionaban para el aula: formatos clásicos y escolarizados en los que no se asignaba a los estudiantes un rol protágonico en la construcción de conocimientos, ni orientado a la acción. En un sentido similar, pero refiriéndose a las nociones de salud y enfermedad, Revel Chion (2013) plantea: "Los debates referidos a los alcances de los diferentes modelos de salud y enfermedad y a las relaciones entre ellos tienen lugar en ámbitos muy alejados de las instituciones educativas, ya sean estos ámbitos filosóficos, epidemiológicos o históricos; por ello, esos debates muchas veces llegan a las instituciones educativas con años de retraso. Es así como, al menos en educación, coexisten modelos ya perimidos con otros más recientes, siendo todos relativamente insuficientes para explicar fenómenos tan complejos como los relacionados con la salud y la enfermedad a la luz de las demandas curriculares actuales..."

Los resultados de investigación identificados orientaron el desarrollo de una propuesta teórica de referencia y nos llevaron a plantear la necesidad de diseñar algunos ejemplos de aplicación y estrategias específicas de acompañamiento a los profesores para favorecer el desarrollo de competencias que les permitan abordar la complejidad de los asuntos socio-científicos en sus clases. 


\section{¿Cómo construir modelos interdisciplinarios "racionales y razonables" para interpretar y abordar fenómenos complejos en las aulas?}

En relación a la propuesta de referencia que mencionábamos, se trata de una propuesta de criterios, fundamentada teóricamente y enmarcada en el modelo cognitivo de Ciencia Escolar (Izquierdo, et al., 1999), que orienta la construcción de modelos interdisciplinarios diseñados ad hoc para interpretar y abordar fenómenos complejos (Bahamonde, 2007; Bahamonde y Pujol, 2009). Consideramos que esta propuesta puede constituir un aporte para la fundamentación, el diseño y la implementación de proyectos educativos, de estrategias de desarrollo profesional docente y de materiales curriculares.

La alimentación humana constituyó, desde mi punto de vista, un ejemplo paradigmático a partir del cual desarrollar la propuesta. La elección del ejemplo no fue casual, ya que la alimentación es una de las numerosas actividades que configuran la vida cotidiana de cualquier grupo social, del presente o del pasado. Por su especificidad y polivalencia adquiere un lugar central en la caracterización de la especie humana. Se trata de un fenómeno complejo, que integra estrechamente las dimensiones biológica y sociocultural, al punto de condicionarlas mutuamente. Estas dimensiones son dinámicas y cambian con el paso del tiempo. Para su abordaje es ineludible una aproximación abarcadora, que visualice el estudio de la alimentación, no sólo desde el punto de vista biológico y nutricional, sino como la expresión de una cultura. Por esta razón, los modelos científicos escolares irreductibles, por ejemplo, el de Ser Vivo, de Célula o de Cambio Químico, resultan perspectivas parciales, necesarias pero insuficientes, a la hora de modelizar este concepto intrínsecamente humano. En una línea similar de argumentación Rivarosa y De Longhi (2013) plantean: "Habitualmente el estudio de esta noción (alimentación) en la escuela se reduce en su dimensión conceptual a los aspectos bio-estructurales-funcionales, sin contemplar que, inserta en la programación escolar, está transversalizada por las prácticas culturales familia-sociedad, economía y tradición, consumo y salud. Por ello, su enseñanza debería incluir necesariamente el ámbito del aprendizaje social, es decir al ser y saber convivir".

Surge entonces, la necesidad de utilizar un enfoque interdisciplinar y multidimensional, que pueda orientar una transposición didáctica por parte de los docentes, ajustada al proyecto y al contexto educativo, sin renunciar al pensamiento teórico.
Para ello recurrimos a la metáfora planteada por Fourez (1997), proponiendo la construcción de un islote interdisciplinario de racionalidad alrededor del concepto de alimentación humana. El islote o modelo ad hoc, se construye a partir de conceptualizaciones que provienen de los modelos explicativos de distintas áreas disciplinares escolares y de los modelos cotidianos. Estas conceptualizaciones se recombinan para explicar dicho concepto o buscar soluciones a problemas significativos para los alumnos, en función de los objetivos del proyecto educativo específico. Su construcción pone en juego nociones como, por ejemplo, cultura, sociedad, producción, consumo, objeto y proceso tecnológico, en interacción con las propias de los campos de la biología y la salud. La nueva conceptualización es superadora de las visiones disciplinares clásicas, porque dota de la capacidad para comunicarse y discutir racionalmente. Esto significa que en esas discusiones la realidad va a ser interpretada en función del modelo teórico acordado y construido ad hoc. Sin embargo, es importante señalar que este diálogo interdisciplinar, alrededor de un problema u objeto de estudio debe asumir el reto de un ir y venir entre la óptica disciplinar y la global, para evitar reduccionismos y permitir la traducción de un marco de referencia a otro, sin perder su potencialidad explicativa.

En relación al abordaje multidimensional, debería tomar en cuenta diversas prácticas sociales de referencia, que den lugar a acciones o actividades de carácter científico, tecnológico y sociocultural por ejemplo, de producción artesanal o industrial, de investigación, actividades domésticas e incorporar variedad de perfiles sociales, como por ejemplo, antropólogos o nutricionistas, pero también cocineros, productores de alimentos, amas de casa, entre otros.

Se trata en definitiva de un recorte curricular multirreferenciado en un sentido similar a la propuesta de Astolfi (1998) sobre la alimentación humana, pero que podría pensarse en relación a otras problemáticas de características equivalentes.

Por último, una condición ineludible es que el islote sea razonable para los alumnos, en la medida que sea plausible y significativo para ellos y que sea un conocimiento orientado a la acción (Izquierdo, 2005). Por esta razón, en el marco del modelo cognitivo de ciencia escolar, es necesario que los problemas o situaciones definidos sean reales, motivadores, cercanos a los alumnos y que conecten con sus modelos de conocimiento cotidiano (Bahamonde, 2007; Bahamonde y Pujol, 2009). 


\section{Propuestas basadas en la investigación y fundamentadas teóricamente para abordar la modelización de temas complejos.}

\section{Ejemplo I \\ El caso de la alimentación humana}

Toscano y colaboradores (1994) y Pujol (2003), entre otros autores plantean ya desde hace tiempo la necesidad de concebir la alimentación humana, como conocimiento escolar, de manera amplia.

En el mismo sentido, hemos desarrolado una propuestas teórica actualizada sobre la alimentación humana como un recorte curricular multirreferenciado, a partir de la construcción de islotes interdisciplinarios de racionalidad y razonabilidad (Bahamonde 2007, Bahamonde y Pujol, 2009), en el marco de la ciencia escolar (Izquierdo et al., 1999). La construcción de estos islotes o modelos teóricos ad hoc permite hacer frente a la complejidad de la enseñanza de asuntos socio-científicos, como la alimentación, dando lugar a la concreción de un nuevo campo teórico estructurante de la ciencia escolar, es decir, un espacio de problemas con personalidad propia y alta densidad conceptual (Adúriz Bravo, 2001). Esto es así porque los modelos científicos escolares que reproducen lo propio de las disciplinas científicas resultan perspectivas parciales para modelizar estas ideas. Nuevas investigaciones en el ámbito de la didáctica de la ciencias y de la biología refuerzan estas ideas (Bahamonde y Diaco, 2013; Rivarosa y De Longhi, 2012; Revel Chion et al., 2013).

Tomando como base la propuesta teórica descrita elaboramos desde el Ministerio de Educación de Argentina y la FAO (2006-2009) un proyecto de desarrollo profesional para facilitar la evolución de los modelos iniciales de los docentes sobre la enseñanza de este tema hacia modelos actualizados y basados en la investigación, sobre el diseño de unidades didácticas- Pueden verse detalles en http:// www.alimentosargentinos.gov.ar/contenido/revista/ediciones/47/articulos/r47_03_FAO.pdf ). En el marco del mismo proyecto, se desarrolló un conjunto de materiales educativos que integran propuestas de enseñanza para los docentes y actividades para los chicos. Estos materiales son de uso libre y descargables desde la web (Proyecto de Educación Alimentaria y Nutricional, Ministerio de Educación de Argentina y FAO, 2009:

http://coleccion.educ.ar/coleccion/CD23/contenidos/ escuela/textos/index13.html).
Enmarcado en la misma perspectiva teórica pero dirigido a la divulgación científica para todos los públicos, estamos desarrollando actualmente el Proyecto: La alimentación, potencial escenario para el debate y la educación científica y cultural, financiado por el Consejo Nacional de Investigaciones Científicas y Tecnológicas de Argentina. Como parte de las actividades de dicho proyecto coordiné el diseño y puesta en marcha de un Observatorio de Educación Alimentaria online www.odeea.com

El observatorio se pensó como un centro de referencia para contribuir a la creación de conciencia social sobre la importancia de la alimentación humana, como hecho multidimensional y complejo y como factor con gran potencial innovador y educativo.

Ofrece a los usuarios una base de datos y recursos actualizada, dando a conocer materiales y actividades desarrollados en el proyecto. Entre los recursos, el sitio cuenta con una selección de cine, publicidad, literatura, museos, programas educativos y guías de análisis para cada rubro, vinculados con la temática. Entre las actividades llevadas a cabo se incluyen el Concurso Fotográfico Fotobocado: Escenas y prácticas de alimentación y la Campaña Publicitaria: Volvamos a comer.

El observatorio se propone también crear espacios de comunicación e intercambio que habiliten nuevas perspectivas en la conceptualización de la temática.

\section{Ejemplo II \\ Biología Molecular y Genética, nuevas agendas científicas y aplicaciones tecnológicas}

Tomando como punto de partida el enfoque teórico de referencia, elaboramos una unidad didáctica para abordar en la educación secundaria, temáticas de fuerte relevancia e impacto social, vinculadas a la nueva Biología (Bahamonde y Rodríguez, 2013). Se diseñaron dos casos que problematizan la clonación exitosa de mamíferos y sus implicancias a nivel terapeútico y reproductivo y la contribución de las tecnologías del ADN en el esclarecimiento de delitos de apropiación ilegal de niños en la última dictadura militar.

Los casos elegidos, funcionan como epítomes o ejemplares que nos permiten abordar lo que venimos denominando asuntos socio-científicos, ya que se trata de temas que se prestan al debate público por sus muchas implicancias sociológicas, religiosas, políticas, filósoficas, económicas, médicas, ecológicas, pero principalmente éticas. Son temas que requieren de discusiones y negociaciones

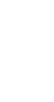


colectivas, que habiliten la construcción de consensos y la toma de decisiones responsables y situadas. Claramente involucran conocimiento tecnocientífico, y al mismo tiempo lo exceden, obligándonos a considerar distintas perspectivas en su abordaje. En este sentido podemos caracterizarlos también como problemas multidimensionales complejos que deberían acercar la representaciones sociales y conocimientos cotidianos de los estudiantes con las conceptualizaciones vinculadas a los modelos explicativos de distintas áreas disciplinares. De ahí la inclusión de estos conocimientos y debates en la escuela, por su potente carácter educativo, que brinda oportunidades a los estudiantes de discutir problemas auténticos con base científica, desde una mirada compleja. Dicha mirada no pretende eludir cuestiones controversiales, ni fomentar posturas ilusorias acerca de la neutralidad de la ciencia o de su capacidad a priori, por su carácter de conocimiento unificado, para resolver los problemas que nos atañen a todos como ciudadanos.

El diseño de la unidad didáctica se enmarca en el Modelo Cognitivo de Ciencia Escolar (Izquierdo et al., 1999), tributario de una concepción modelo-teórica de la ciencia (Giere, 1999). Las actividades elaboradas convierten en objeto de problematización y reflexión noticias de divulgación científica que circulan en los medios de comunicación, así como películas de ficción y hechos de nuestra historia reciente subrogados por modelos biológicos estructurantes (modelo y tecnologías del ADN). De este modo, los modelos biológicos se vinculan con los modelos mentales que construyen los alumnos para dotar de nuevos significados a diferentes parcelas de la realidad que han sido problematizadas y sobre las que desea intervenir (Izquierdo, 2000).

Por otra parte, la búsqueda de explicaciones o soluciones a las situaciones o problemas planteados, conduce a la necesidad de trabajar ciertos conceptos, modelos o teorías científicas, y no otras. En esta propuesta se trabajan nociones científicas del campo conceptual de la Biología Molecular y la Genética vinculadas al modelo de ADN y de las tecnologías vinculadas . Las actividades sugeridas incluyen también los antecedentes que preceden su desciframiento y los consecuentes, entre ellos, las aplicaciones tecnológicas derivadas de esos conocimientos, que han dado lugar a nuevos problemas de investigación y han producido un enorme impacto en nuestras sociedades. El abordaje de este tema brinda la posibilidad de reconstruir, en paralelo con la construcción de nociones científicas, parte del proceso histórico que dio origen a los conceptos estudiados valiéndonos de contenidos de la epistemología del siglo XX, ambientados en la historia de la ciencia e informados por la sociología de la ciencia (Adúriz-Bravo, 2005), a partir del trabajo con diferentes fuentes (videos, textos, líneas de tiempo, simulaciones, entre otros). Esta forma de presentar las ideas científicas arraigadas en los escenarios y los protagonistas de su construcción, apunta a la inclusión de una reflexión contextuada acerca de la actividad científica, como actividad humana, colectiva, situada. Favorece que los estudiantes "puedan entender su naturaleza, significado y sentido al determinar las causas que posibilitaron su aparición e identificar las diferentes etapas de su construcción en el ámbito científico" Adúriz-Bravo (2010).

\section{A modo de cierre}

En este artículo me propuse presentar un recorrido que, partiendo de las demandas sociales actuales a la educación científica con foco en la biología y los desafíos que implica vinculados con la formación de ciudadanía de pleno derecho, desarrolla una propuesta teórica para la modelización de temas complejos relevantes para los estudiantes y la sociedad y propone diferentes ejemplos de aplicación para los ámbitos de la educación primaria y secundaria y de la divulgación científica, así como estrategias de acompañamiento a los profesores para favorecer el desarrollo de competencias que les permitan abordar la complejidad de los asuntos socio-científicos en sus clases.

\section{Bibliografía}

Abell, S.K. (ed.). (2000). Science teacher education: An international perspective. Dordrecht: Kluwer Academic Publishers.

Acevedo Díaz, J.A. (2010). La evaluación PISA de la alfabetización científica y la didáctica de las ciencias. Divulgacióny Cultura Científica Iberoamericana. http:// www.researchgate.net/publication/261027233_La_ evaluacin_PISA_de_la_alfabetizacin_cientfica_y_la_ didctica_de_las_ciencias

Acevedo Díaz, J.A. (2004). Reflexiones sobre las finalidades de la enseñanza de las

ciencias: Educación científica para la ciudadanía. Revista Eureka sobre Enseñanza y

Divulgación de las Ciencias, 1(1), 3-16.

Adúriz-Bravo, A. (2010). Aproximaciones histórico-epistemológicas para la enseñanza de conceptos disciplinares. Revista EDUCyT, 1 (1): 107-126.

Adúriz-Bravo, A. (2005). Una introducción a la naturaleza de la ciencia: La epistemología en la enseñanza de las ciencias naturales. Buenos Aires: Fondo de Cultura Económica. 
Aikenhead, G.S. (2011). Preface. In T.D. Sadler et al. (Eds.), Socio-scientific issues in the classroom. Teaching, learning and research. Springer.

Anadón, M., Bahamonde, N. y Lozano, E. (2013). El desarrollo profesional docente de profesores de ciencias básicas en el marco de la conformación de una comunidad de práctica y aprendizaje: avances de investigación. VI Congreso Nacional y IV Internacional de Investigación Educativa. Neuquén.

Arca, M. y Guidoni, P. (1989). Modelos infantiles y modelos científicos sobre la morfología de los seres vivos. Enseñanza de las Ciencias, 7(2), 162-167

Astolfi, J. P. (1998). Desarrollar un currículo multirreferenciado para hacer frente a la complejidad de los aprendizajes científicos. Enseñanza de las Ciencias, 16 (3), 375-385.

Bahamonde, N., Lozano, E. Y Diaco, P. (enviado 2014). Una comunidad de aprendizaje para pensar la enseñanza de las ciencias en el ámbito de la universidad. XI Jornadas Nacionales y VI Congreso Internacional en Enseñanza de la Biología.

Bahamonde, N. y Rodríguez, M. (2013). OJO MENDOZA

Bahamonde, N. y Diaco, P. (2013). Modelización del fenómeno de alimentación humana: de la mirada disciplinar a la multirreferencialidad. IX Congreso Internacional sobre Investigación en Didáctica de las Ciencias. Barcelona. pp: 252-258. http://congres. manners.es/congres_ciencia/gestio/creacioCD/cd/ articulos/art_1009.pdf

Bahamonde, N. y Pujol, R.M. (2009). Los modelos de conocimiento científico escolar de un grupo de docentes sobre la alimentación humana. Enseñanza de las Ciencias, Número Extra. VIII Congreso Internacional sobre Investigación en Didáctica de las Ciencias. Barcelona,pp. 2900-2904.

http://ensciencias.uab.es/congreso09/numeroextra/art2900-2904.pdf

Bahamonde, N. y Pujol, R.M. (2009). Un enfoque teórico para el abordaje de temas complejos en el aula: el caso de la alimentación humana. VIII Congreso Internacional sobre Investigación en Didáctica de las Ciencias. Barcelona. España. 2009.

http://ensciencias.uab.es/congreso09/numeroextra/art2315-2319.pdf

Bahamonde, N. (2007). Los modelos de conocimiento científico escolar de un grupo de maestras de educación infantil: un punto de partida para la construcción de "islotes interdisciplinarios de racionalidad" y "razonabilidad" sobre la alimentación humana. Tesis doctoral inédita. Universitat Autònoma de Barcelona. Barcelona.

Christensen, C. y Fensham, P.J. (2012). Risk, Uncertainty and Complexity in Science Education. B.J. Fraser et al. (eds.), Second International Handbook of Science Education, 751. Springer International Handbooks of Education.

Diario Oficial de la Unión Europea (2006). Recomendación del parlamento europeo y del consejo de 18 de diciembre de 2006 sobre las competencias clave para el aprendizaje permanente (2006/962/CE).

Fensham, P. J. (2009) Real world contexts in PISA science: implications for context-based science education. Journal of Research in Science Teaching, 46(8), pp. 884-896.

Fourez, G. (1997). Qu'e entendre par "ilot de rationalité" Et par "ilot interdisciplinaire de rationalité"? Aster 25, 217-225.

http://documents.irevues.inist.fr/bitstream/handle/2042/8686/ASTER_1997_25_217. pdf?sequence $=1$

García, P. (2005). Los modelos como organizadores del currículo en biología. Enseñanza de las Ciencias. Número extra. 1-5.

Giere, R. (1999). Del realismo constructivo al realismo perspectivo. Enseñanza de las Ciencias. Número extra. 9-13.

Gómez Galindo, A.A. (2011). La enseñanza de la Biología en la educación básica: modelización y construcción de explicaciones multimodales. Memorias del I Congreso Nacional de Investigación en Enseñanza de la Biología y la Educación Ambiental. Bio-grafía. Escritos sobre la Biología y su enseñanza. Edición Extra-Ordinaria. 521-532.

Gómez Galindo, A.A. (2009). El estudio de los seres vivos en la Educación Básica. Enseñanza del sistema nervioso desde un enfoque para la evolución de los modelos escolares. Universidad Autónoma de Nuevo León. México.

Gómez Galindo, A.A. (2007) Fundamentación teórica y diseño de una unidad didáctica para la enseñanza del modelo ser vivo en la escuela primaria. Enseñanza de las Ciencias, 25(3), 325-340. http:// www.redalyc.org/articulo.oa?id=27417105

Harlen, W. (2002). Evaluar la alfabetización científica en el programa de la OECD para la evaluación internacio- 
nal de estudiantes (PISA). Enseñanza de las Ciencias, 2002, 20 (2), 209-216.

Izquierdo, M. (2005). Hacia una teoría de los contenidos escolares. Enseñanza de las Ciencias, 23 (1): 111-122.

Izquierdo, M. 2000. Fundamentos epistemológicos. En Perales. F. J. Y Cañal de León, P. (Directores): Didáctica de las ciencias experimentales.

Izquierdo, M., Espinet, M., García, M. P., Pujol, M. R. Y Sanmartí, N. (1999). Caracterización y fundamentación de la ciencia escolar. Enseñanza de las Ciencias. Número extra. 79-92.

Jiménez Aleixandre, M. P. y Federico-Agraso, M. (2009). Justification and persuasion about cloning. Research in Science Education, 39 (3): 331-347.

Legardez, A. y Simonneaux (dir.) (2006). L'école à l'épreuve de l'actualité : Enseigner les questions vives. Issy-les-Moulineaux : ESF.

Lemke, J. L. (1997). Aprender a hablar ciencia.Lenguaje, aprendizaje y valores. Paidós. Barcelona.

Ministerio de Educación. República Argentina (2012). Núcleos de Aprendizaje Prioritarios. Campo de Formación General. Educación Secundaria Orientada. Resolución CFE N 180/12.

Ministerio de Educación. República Argentina. (2011). Marcos de Referencia. Educación Secundaria Orientada. Bachiller en Ciencias Naturales. Resolución CFE N 142/11.

OECD (2006). Assessing Scientific, Reading and Mathematical Literacy: A framework for PISA 2006. Paris: OECD.

OECD (2003). Assessment framework mathematics, Reading, Science and problems solving knowledge and skills. OECD.

Roberts, D. (2007). Scientific literacy/science literacy. In S. K. Abell \& N. G. Lederman (Eds.) International handbook of research on science education. 729-780. Mahwah, NJ: Lawrence Erlbaum Associates.

Revel Chion, A. Meinardi, E. y Adúriz Bravo, A. (2013) Elementos para un análisis histórico-epistemológico del concepto de salud con implicaciones para la enseñanza de la Biología. Filosofía e História da Biologia. V 8, n.1-19.
Sanmartí, N. (2005). La unidad didáctica en el paradigma constructivista, en Couso, D. Badillo, E., Perafán, G. y Adúriz-Bravo, A. (comps.). Unidades didácticas en ciencias y matemáticas. 13-58. Bogotá: Cooperativa Editorial Magisterio.

Ziedler D. L., Sadler, T. D., Simmons, M. L. and Howes, E.V. (2005). Beyond STS: A Research-Based Framework for Socioscientific Issues Education. Wiley InterScience (www.interscience.wiley.com) 